\title{
Imaging Capabilities, Performance and Applications of the Hard X-ray Nanoprobe Beamline at NSLS-II
}

\author{
H. Yan*, X. Huang, E. Nazaretski, N. Bouet, J. Zhou, W. Xu, and P. Ilinski and Y. S. Chu
} National Synchrotron Light Source II, Brookhaven National Laboratory, Upton, NY 11973, USA

*hyan@bnl.gov

The hard x-ray nanoprobe (HXN) at National Synchrotron Light Source II (NSLS-II) of Brookhaven National Laboratory (BNL), USA, is a scanning microscopy beamline equipped with a versatile instrument satisfying a number of imaging demands. It is designed to offer an initial spatial resolution of $\sim 10 \mathrm{~nm}$ and provides absorption-, phase-, fluorescence- and diffraction-contrasts for mapping elemental, chemical and structural variations at the nanometer scale. The beamline has been in operation since 2015, and routinely provides measurements with this resolution for applications in many areas of science. In this presentation we will discuss imaging capabilities, performance and applications of HXN through a few examples. We will show how multimodal imaging is achieved with a single raster scan.

$\mathrm{HXN}$ is a $120-\mathrm{m}$ long beamline with an operation energy of 6-25 keV, and has two microscopes for user operation. One is equipped with multilayer Laue lenses (MLL) for high resolution applications $(\sim 10 \mathrm{~nm}$, $\geq 12 \mathrm{keV}$ ) and the other one with Fresnel zone plates (FZP) for modest resolution applications (30-100 $\mathrm{nm}, \leq 12 \mathrm{keV})$. The MLL microscope has a much shorter working distance $(\sim 0.5 \mathrm{~mm})$ than that of the FZP microscope ( $>5 \mathrm{~mm})$, due to MLL's high numerical aperture ( $\sim 5 \mathrm{mrad})$ and relatively small aperture size $(\sim 50 \mu \mathrm{m})$. In addition to an $\mathrm{x}-\mathrm{y}-\mathrm{z}$ scanner for sample scanning, both microscopes have a rotational stage that enables tomography and diffraction capabilities. The FZP microscope has an additional parallel kinematic device with six degrees of freedom to further increase the accessible reciprocal space for diffraction. Both microscopes are installed inside the vacuum chamber, and can work under ambient, vacuum and helium conditions. In most cases we operate under helium conditions in order to reach thermal equilibrium faster [1].

At each scanned position, fluorescence, transmission and diffraction (for crystalline samples) signals can be collected simultaneously. This enables multimodal imaging capability with a single raster scan. A 3element Vortex detector placed at 90 degrees with respect to the beam is used to collect fluorescence spectra, and two pixel-array detectors (Merlin, $55 \mu \mathrm{m}$ pixel size, 516 x 516 pixels) for recording transmission and diffraction patterns, respectively. Elemental maps are obtained by fitting the fluorescence spectra and morphological images are acquired by analyzing the transmission patterns either based on deterministic differential-phase-contrast or ptychography reconstruction. Software packages with CPU/GPU acceleration have been developed to enable fast data analysis on-site. For crystalline samples, the diffraction pattern is analyzed using either conventional reciprocal space mapping (RSM) methods to yield a nanodiffraction topography or more advanced Bragg ptychography reconstruction technique for quantitative strain mapping $[2,3]$.

We present resolution characterization results with MLL optics. Because MLL is a 1D focusing optic and requires additional angular motion for Bragg condition, the alignment of a MLL microscope poses a challenge. Small amount of misalignment in both the azimuthal angle that determines the orthogonality and the separation distance between two MLLs can have a profound impact on the focusing performance of the system and leads to considerable aberration [4]. Using a set of MLLs with diffraction-limited focus size of 10-nm, we show a full-width-at-half-maximum (FWHM) focus size of $15.3 \times 16.9 \mathrm{~nm}^{2} \mathrm{using}$ 
conventional knife-edge scans, while the ptychography reconstruction produced a FWHM size of $13.9 \mathrm{x}$ $12.3 \mathrm{~nm}^{2}$. Power-spectrum-density (PSD) analysis of a test pattern fluorescence image revealed the smallest detectable feature size down to $10.3 \times 10.8 \mathrm{~nm}^{2}$. With the aid of ptychography reconstruction, the resolution can be further improved below $10 \mathrm{~nm}$ [5]. Combining the very small focus size and the multimodal imaging capability, HXN provides a unique tool for a comprehensive investigation of material properties at a nanometer scale. As an example, in Fig. 1 we depict fluorescence, differential-phase, integrated phase from DPC and reconstructed phase from ptychography algorithm images of a cube nanoparticle array. Nanoparticles have a monodispersive size of $50 \mathrm{~nm}$ and the gap between them is as small as $10 \mathrm{~nm}$. With a single raster scan, we attain both elemental and morphological information. In the reconstructed phase image, the gap can be well resolved, indicating a resolution below $10 \mathrm{~nm}$. A PSD analysis (Fig. 1f) confirms this observation. Two dimensional (2D) fly-scan fluorescence mapping with a minimum dwell time of $20 \mathrm{~ms}$, DPC and ptychography reconstruction are basic capabilities of HXN and are provided to users as routine measurements in daily operation.

Tomography and nanodiffraction are routinely done at HXN as well, and their demands are increasing. A few application examples will be presented to discuss our current capabilities and limitations. Looking ahead, we are continuously improving the resolution. Using a wedged MLL fabricated at BNL, recently we achieved a clean focus of $8 \mathrm{~nm}$ at $15 \mathrm{keV}$ that is suitable for scanning applications. We envision that multimodal imaging capability with below $10 \mathrm{~nm}$ resolution will be available to users at HXN in the not too distant future. We are also pushing high-resolution (below $10 \mathrm{~nm}$ voxel size) tomography. Moreover, we are developing depth-resolved ptychography [6] and Bragg ptychography for quantitative strain imaging. The ultimate goal of the beamline is to enable single digit resolution in 3D for in-situ/in-operando elemental, chemical and structural imaging.

References:

[1] E. Nazaretski et al. J. Sync. Rad. 24, 1113 (2017)

[2] H. Yan et al. Optics Express 25 (2017) 25234.

[3] M. J. Highland et al. Applied Physics Letters 111 (2017) 161602.

[4] M. O. Hill et al. Nano Letters 18 (2018) 811.

[5] H. Yan et al. Nano Futures 2 (2018) 011001.

[6] H. Öztürk et al. Optica 5 (2018) 601
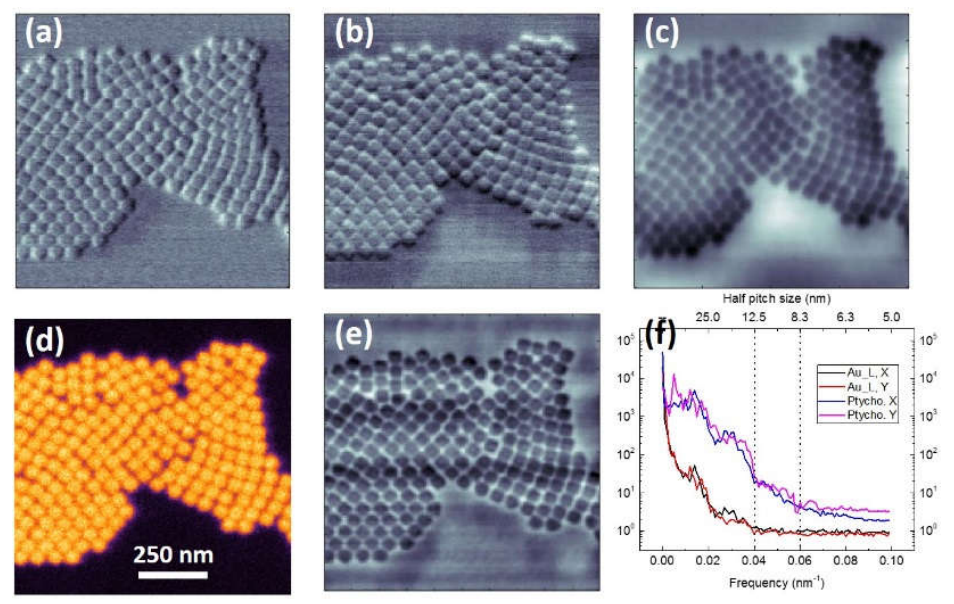

Figure 1 X-ray images of an $\mathrm{Au}$ nanoparticle array obtained from a single raster-scan. (a) differential phase gradient horizontal, (b) differential phase gradient vertical, (c) integrated phase from (a) and (b), (d) Au fluorescence and (e) reconstructed phase from ptychography phase-retrieval algorithm. The particle has a cube shape with a size of $50 \mathrm{~nm}$ and separation distances as small as $10 \mathrm{~nm}$. (f) is PSD plot of (d) and (e), which indicates a detectable feature size of $12.5 \mathrm{~nm}$ in the fluorescence image and $8 \mathrm{~nm}$ in the phase image. 